Théologiques

Théologiques

\title{
La praxéologie pastorale : faire théologie selon un paradigme praxéologique
}

\section{Jean-Guy Nadeau}

Volume 1, numéro 1, mars 1993

Théologie et sciences humaines en contexte universitaire

URI : https://id.erudit.org/iderudit/602383ar

DOI : https://doi.org/10.7202/602383ar

Aller au sommaire du numéro

\section{Éditeur(s)}

Faculté de théologie de l'Université de Montréal

ISSN

1188-7109 (imprimé)

1492-1413 (numérique)

Découvrir la revue

Citer cet article

Nadeau, J.-G. (1993). La praxéologie pastorale : faire théologie selon un paradigme praxéologique. Théologiques, 1(1), 79-100.

https://doi.org/10.7202/602383ar
Résumé de l'article

La praxéologie pastorale situe d'emblée l'élaboration théologique dans un paradigme praxéologique de recherche-action-formation dont la pratique est le point de départ et le point d'arrivée. Dans un premier temps, l'auteur situe la praxéologie pastorale en rapport avec la praxéologie, la théologie et la théologie pratique. Dans un second temps, il présente la démarche, ou la méthodologie, de praxéologie pastorale où se déploient ces rapports. La praxéologie y apparaît comme un effort d'herméneutique pratique articulant les tâches universitaires de recherche, de critique et de formation professionnelle menées en contexte pluridisciplinaire. 


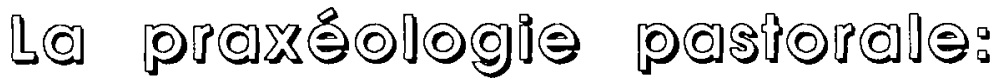 ใด

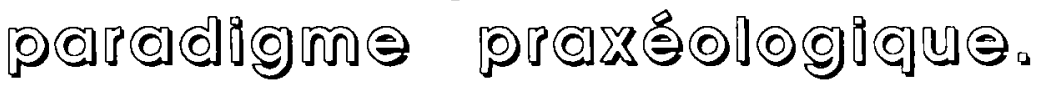

Jean-Guy NADEAU

Faculté de théologie Université de Montréal

\section{RÉSUMÉ}

La praxéologie pastorale situe d'emblée l'élaboration théologique dans un paradigme praxéologique de recherche-action-formation dont la pratique est le point de départ et le point d'arrivée. Dans un premier temps, l'auteur situe la praxéologie pastorale en rapport avec la praxéologie, la théologie et la théologie pratique. Dans un second temps, il présente la démarche, ou la méthodologie, de praxéologie pastorale où se déploient ces rapports. La praxéologie y apparaît comme un effort d'herméneutique pratique articulant les tâches universitaires de recherche, de critique et de formation professionnelle menées en contexte pluridisciplinaire.

Étudiant au début des années 1970, je faisais de l'auto-stop pour revenir de mes cours à l'université. Aux questions des chauffeurs, je répondais que j'étudiais en études pastorales, précisant, avec humour, que c'était le département des ventes de la théologie. Je confondais alors les études pastorales avec la pastorale que je tenais, naïvement mais traditionnellement, pour l'application de la théologie, la mise en marché de la foi chrétienne. Mon expérience a modifié ces conceptions et la pastorale m'apparaît maintenant comme un lieu d'élaboration chrétienne et théologique, et les études pastorales, dont relève 
initialement la praxéologie pastorale, comme l'accompagnement critique de cette élaboration sur le terrain comme à l'université.

Je présenterai ici la praxéologie pastorale en la situant en regard de la théologie et des sciences sociales. La praxéologie pastorale s'identifie comme une approche herméneutique des pratiques chrétiennes, visant à intégrer analyse empirique et discours critique. Science de l'action sensée, elle vise à faire émerger à la conscience la réalité et le discours d'une pratique particulière pour les confronter à ses porteurs et à ses référents, de façon à rendre cette pratique plus consciente de son langage, de ses modes et de ses enjeux en vue d'accroître sa pertinence et son efficacité, son service ou son coefficient de libération. La praxéologie pastorale apparaît ainsi non seulement comme une pratique de recherche intellectuelle, mais d'abord comme une pratique de responsabilisation des sujets de l'action.

Pastorale, elle s'intéresse à des pratiques préoccupées par les enjeux fondamentaux de l'existence humaine, animées entre autres par la reconnaissance de Jésus Christ et le façonnement du Royaume de Dieu. Son défi: articuler critiquement logos de l'action, logos de la culture et logos de la foi; mémoire, promesse et action dans le présent. La requête d'une corrélation mutuellement critique entre l'expérience et le langage communs d'une part et la tradition chrétienne d'autre part (Tracy 1975), ou "entre la théorie el la praxis interprétées du fait chrétien et la théorie et la praxis interprétées de la situation contemporaine" (Tracy 1983: 76), marquait déjà les débuts de la praxéologie pastorale (Lucier 1972).

Cette science théologique s'adresse d'abord à des gens d'action: pasteurs, agents de pastorale laïques, chrétiens et chrétiennes qui entendent améliorer leur pratique en termes de "signifiance" et d'efficacité, en fonction d'une double fidélité/pertinence quant au monde contemporain et au Règne de Dieu avec leurs défis, requêtes et possibilités. Ces agents se trouvent ainsi confrontés à retisser les liens entre l'existence quotidienne, l'action et l'intelligence chrétiennes. En ce sens, elle ne vise pas d'abord l'apprentissage de techniques d'action pastorale, mais l'étude de cette action dans une approche résolument herméneutique.

Signalons quelques éléments majeurs qui ont marqué l'émergence de la praxéologie pastorale à la fin des années 1960 (Nadeau 1990: 139142). 
1) Un souci de pertinence de la réflexion théologique et de l'agir pastoral vis-à-vis du réel, ce qui a conduit la praxéologie pastorale à une écoute rigoureuse de ce réel.

2) Un intérêt pour la signification et l'efficacité des pratiques pastorales, situant l'acte théologique "au point de jonction des impératifs du sens et des impératifs de l'action" (Lucier 1974: 7).

3) La conscience que les pratiques pastorales sont d'abord des pratiques, et donc à étudier comme telles, qui a tiré la praxéologie pastorale du côté des sciences de l'action.

4) Une lecture de l'Évangile comme récit interprétant de la pratique et de la cause de Jésus Christ, nécessaire pour saisir, évaluer et relancer les prétentions pastorales de nos pratiques. La quête de la pertinence de cette mémoire pour les hommes et les femmes de ce temps fonde le projet de praxéologie pastorale, comme celui de toute théologie, d'ailleurs. Avec les requêtes de connaissance et de reconnaissance, de sens, de planification et de compétence qui marquent toute pratique, c'est cette option de foi qui nous convie à la tâche de corrélations critiques entre l'herméneutique de telle pratique dans son milieu et l'herméneutique de la tradition dont elle se réclame.

5) La reconnaissance du caractère déterminant des pratiques dans l'élaboration de l'humain et de l'histoire, et celle du sujet comme sujet social, agissant et souffrant, interprétant, éthique, etc., qui ont mené la praxéologie pastorale à une reconnaissance de la responsabilité interactive des acteurs d'une pratique.

La praxéologie pastorale veut donc articuler l'étude des pratiques (praxéologie), en regard de leurs référents chrétiens (pastorale). Alors que la première dimension appelle un rapport étroit aux sciences sociales, particulièrement aux sciences de l'action, la seconde appelle un rapport étroit à la théologie, éthique et herméneutique articulant ces deux dimensions

\section{Une science de la pratique}

C'est dans un souci de retrouver la force élaborante de l'action pour la théologie et de distinguer son approche d'une théologie pratique appliquée, qu'une équipe de la Faculté de théologie de l'Université de Montréal a forgé le vocable de praxéologie pastorale, inscrivant d'emblée sa démarche dans une approche praxique et multidisciplinaire. 


\subsection{Praxéologle pastorale et praxéologie}

La praxéologie relève d'abord des sciences humaines et sociales: sciences économiques, sociologie du travail, sciences de l'organisation et de la décision, etc. Le terme est issu de la philosophie des sciences où il désignait les sciences des fonctions (Bourdeau 1882). II a été repris en philosophie de l'action pour désigner une sagesse pratique des principes de l'action humaine et de ses techniques (Espinas 1897). Kotarbinski (1937, 1965) et Von Mises (1950) ont développé cette sagesse en une analytique de l'action et de ses agents visant les principes généraux et une méthodologie de l'action efficace, voire de l'efficacité de l'action.

De ces derniers, la praxéologie pastorale a d'abord retenu le vocable de praxéologie et son objet spécifique: l'action efficace. De von Mises, elle retient aussi que l'action existe par l'échange entre l'Ego et l'Alter Ego, et qu'il importe d'étudier les problèmes de cet échange. La notion d'interaction, et donc de communication, se tient ainsi au coeur du "paradigme praxéologique" qui intègre "autoposition des acteurs et transformation des situations sociales" (Goyette et Lessard-Hébert 1987: 118).

La praxéologie pastorale se démarque cependant du type de formalisation de ces praxéologies et de leur analyse de l'action réduite à "l'ajustement des moyens à la réalisation d'une fin donnée" (Daval 1963:139). Préoccupées de la signification et de la pertinence aussi bien que de l'efficacité des pratiques, les études pastorales ne sauraient endosser une conception aussi réductrice de la science de l'action, bien qu'elles doivent en tenir compte. On ne saurait ici séparer l'éthique de la logique (Kaufmann 1968: 11). D'où l'intégration à la praxéologie pastorale des dimensions éthiques et herméneutiques de l'action. Avec le souffle qui le caractérise, Jacques Grand'Maison, un des initiateurs de la praéxologie pastorale, la présentait comme une démarche de recherche-action "capable d'articuler le vécu, l'action et la pensée, le savoir être, vivre, dire et faire collectif des travailleurs, l'expérience réfléchie, la conscience de classe et la création collectiven (Grand'Maison 1975: 11), et y intégrait la perspective marxienne et la critique des idéologies (Grand'Maison 1974: 14). Sensible aux acteurs d'une pratique, la praxéologie pastorale est alors particulièrement sensible aux liens entre action, intérêt et connaissance. 
La praxéologie pastorale tire donc les sciences de l'action du côté de la pratique, élargissant ainsi leur visée. Elle s'élabore alors comme une sémantique et une pragmatique des pratiques pastorales, ou des pratiques sociales, et l'acte théologique s'y profile d'abord comme émergeant de la compréhension critique, corrélée avec leur tradition, que des acteurs ont de leur pratique.

\subsection{La pratique ${ }^{1}$}

C'est à dessein que, à revers de la tradition marxienne, nous utilisons le terme pratique, appartenant au vocabulaire familier, plutôt que celui de praxis ${ }^{2}$, plus technique et plus noble. La pratique désigne des activités volontaires visant des résultats concrets, une manière concrète d'exercer une activité, voire une manière habituelle d'agir (Le Petit Robert 1978), ou encore, une action complexe régie par des préceptes de toutes sortes (Ricoeur 1989a: 15) et marquée par une certaine durée. Autant d'énoncés contribuant à spécifier la praxéologie pastorale dans le champ théologique.

Au plan systémique, la pratique apparaît comme un système complexe d'actions et d'interactions, orienté vers une fin, comportant des relations de coordination et de subordination, réglé par des règles de différents types. Ce système est situé dans un contexte (institutionnel, socio-culturel) qui l'influence, lui donne signification et est influencé par lui. Cet aspect systémique constitue un premier objet, le plus formel, de la praxéologie pastorale.

A travers ces dimensions systémiques, émerge ..ou se perd, selon les analyses (Ricoeur 1990: 73ss)-- le sujet dont la pratique porte la visée et auquel elle permet de s'inscrire dans le monde. La pratique est alors "un acte d'interprétation que chacun exerce en première personne en se situant, par rapport au monde, dans la perspective d'un certain projet de sens." (Jervolino 1991: 228). Elle

1 Pour un plus ample développement de ces perspectives, cf. Nadeau 1993.

2 Michael Scanlon (1988:81) signalait le refus des théologiens américains de traduire le terme praxis, alors que Paul Ricoeur nous communiquait une résistance semblable, tout en situant les pratiques sur l'échelle de la praxis (1989a:15). Pour David Tracy, "praxis is correctly understood as the critical relationship between theory and practice whereby each is dialectically influenced and transformed by the other" (1975: 243); ce qui mènerait à considérer la praxéologie comme l'élaboration de ce rapport. 
apparaît ainsi en rapport intrinsèque avec la théorie, d'où l'intérêt de l'appellation "praxéologie" pastorale.

Inscription d'un sujet avec d'autres sujets, la pratique est communicationnelle. En plus d'être expression du sujet, elle est échange d'informations, de demandes et de réponses, de services, de possibilités d'être. Dans la mesure où elle est porteuse d'expression de soi, elle est aussi demande d'être reconnu... et lieu d'échange de la reconnaissance. Ainsi est-elle échange, risqué, de sujets.

Interactive, elle est processus d'influence sur les autres, aussi bien que sur le monde, et ainsi marquée d'une foncière dissymétrie agent/patient (Ricoeur 1989a: 24). Agir c'est intervenir sur et non seulement avec, quoiqu'il en soit de notre idéologie. Or, une telle relation dissymétrique fait souvent problème en pastorale, tout comme l'enchevêtrement des lois du système et de l'initiative du sujet. L'inscription responsable du sujet s'affirme alors comme un objet majeur de la praxéologie pastorale.

La pratique laisse des marques sur l'espace social et sur l'histoire aussi bien que sur les personnes. Par ces marques, qui échappent largement à ses acteurs, la pratique acquiert une certaine autonomie. Elle relève ainsi de l'oeuvre, au sens d'une inscription événementielle et historique débordant l'intention de ses acteurs. A cause, certes, de sa situation contextuelle et de ses enchevêtrements, mais à cause aussi de l'autonomisation de l'action que Ricoeur (1971) considère selon le modèle de l'autonomisation du texte. Comme le texte, la pratique s'ouvre ainsi à quiconque sait lire, peut en recevoir des interprétations différentes et même distinctes de celles qu'en font ou en faisaient ses acteurs. On dira alors de la compréhension d'une pratique ce que Ricoeur affirmait de la compréhension d'un texte: "ce qui est à comprendre dans un texte, ce n'est ni son auteur et son intention présumée, ni même la structure ou les structures immanentes au texte, mais la sorte de monde visé hors du texte comme la référence du texte" (Ricoeur 1977b: 38). L'importance et la signification d'une pratique dépassent donc sa pertinence et sa signification initiales, auxquelles s'intéresse d'abord la praxéologie pastorale. Processus de dévoilement aussi bien que de transformation, la pratique comme oeuvre ouvre des possibilités d'être qui peuvent être actualisées dans des situations autres. N'est-ce pas ainsi que la théologie traite la pratique de Jésus, tentant toujours d'en réactualiser à neuf l'évangile? 
La praxéologie pastorale est donc attentive aux pratiques dans leurs aspects structurels, énonciatifs et révélateurs, respectivement travaillés par une sémantique, une pragmatique, une phénoménologie et une herméneutique de l'action et de la pratique (Ricoeur 1989b: 275; 1990). Pastorale, elle porte une attention particulière aux modalités et aux référents propres de l'inscription croyante.

\section{Une herméneutique des pratiques chrétiennes}

\subsection{Praxéologie pastorale et théologie}

$\mathrm{Si}$ on définit la théologie comme fides quaerens intellectum, on pourra identifier la praxéologie pastorale comme praxis fidei quaerens intellectum. Pléonasme dans la mesure où la foi est toujours liée à une pratique, mais nous visons à souligner l'attention prioritaire que la praxéologie pastorale accorde aux pratiques concrètes. Les réflexions de Pierre Lucier, d'abord publiées au début des années 1970, nous permettront de dégager quelques rapports entre praxéologie pastorale et théologie.

"C'est par ses visées les plus fondamentales que la théologie est reliée au monde de l'agir", écrivait Lucier (1974: 27), le champ praxéologique, avec en son centre le geste de Jésus-Christ, constituant le "lieu du Christianisme lui-même" (Lucier 1974: 35). Dans ce cadre praxéologique, la théologie apparaît comme le champ herméneutique, "le cadre de référence utilisé par l'agent pour comprendre son champ d'intervention et son geste même." (Lucier 1974: 19). Renversant la perspective classique, mais non sans connivence avec une conception pratique de la théologie pratique, on pourrait affirmer que c'est la théologie qui se situe dans le champ de la praxéologie pastorale, plutôt que l'inverse, suggéré (ou critiqué) par le titre qu'on m'a proposé pour cet article: "La praxéologie pastorale est-elle un appendice à la théologie?".

Edward Farley a bien montré comment, en se cléricalisant, en se structurant en sous-dișciplines, la théologie s'est éloignée de sa perspective pratique initiale, opérant une dissociation voire une aliénation entre théorie et pratique (Farley 1983a,1983b, 1987). C'est ainsi, affirmait Lucier (1974: 33), que, oubliant leur enracinement pratique et praxéologique, "bien des synthèses théologiques se sont 
avérées inaptes à faire comprendre et à animer une praxis chrétienne de salut: construites à l'abri des sollicitations de la praxis, elles ont péri dans leur isolement et dans leur beauté abstraite, loin des véritables enjeux de l'existence" (Lucier 1974: 33). Si la praxéologie pastorale se présente comme une science du salut, à l'instar de la théologie, on est tenté de préciser: une science de la figuration humaine ou de la pratique de ce salut avec ses sujets et contextes différenciés, ses ressources et possibilités historiques, ses essais concrets, ses effets positifs et négatifs, etc.

Bien qu'ambitieux dans sa visée holistique, le projet de praxéologie pastorale est en même temps plus modeste que ceux des théologies fondamentale et systématique. Centrée sur des pratiques particulières, la praxéologie pastorale ne cherche pas d'abord à élaborer un discours théologique systématique, ni même une théorie de la pratique pastorale, quoiqu'elle s'y attelle aussi. Plus pragmatique, elle vise d'abord l'élaboration d'un discours fécond pour des pratiques particulières. Elle est, à ce titre parmi d'autres, parente d'une theological reflection attentive à dégager d'une pratique ses dimensions d'action, de sens et de responsabilité (Ogletree 1983), et saisie comme un "process centered on discovering one's operative theology as it unfolds in human experience [with an interpretation] which includes both understanding and responsible action or commitment" (Sheehan 1984: 31).

La praxéologie pastorale veut donc revenir aux pratiques, leur redonner place dans l'élaboration théologique. Elle relève ainsi du tournant praxique qui a accompagné le tournant linguistique (Scanlon 1988). Elle considère en effet la pratique comme le point de départ, le fondement et le but de la réflexion théologique, mais sans opposer théorie et pratique ni accorder une primauté absolue à celle-ci. La praxéologie pastorale s'inscrit ainsi dans le modèle de corrélation praxique critique de la typologie des rapports théorie-pratique proposée par Matthew Lamb (1976).

Nous avons signalé que les dynamiques de l'action et de la communication constituent le noeud des sciences de l'action. Elles constituent aussi celui de la science du salut dont l'objet n'est peut-être pas tellement Dieu que son agir et sa communication, comme l'ont saisi les théologies de l'histoire et celles de la Parole, ainsi que les réflexions théologiques sur la Révélation. Structurellement, praxéologie et théologie apparaissent donc fondamentalement liées, bien que distinguées 
par leurs rapports à l'expérience concrète, leurs référents et leurs formalisations.

\subsection{Praxéologle pastorale et théologie pratique}

Si la praxéologie pastorale se distinguait inititalement de la théologie pratique (ou de la théologie pastorale, Lucier 1973: 8), elle paraît aujourd'hui en relever. Dépassant sa perspective déductive et applicative, la théologie pratique a pris ces dernières années un tournant résolument praxique, s'inscrivant de fait dans un paradigme praxéologique. Norbert Mette la définit comme science de l'action (Mette 1979), "science théologique de l'agir à l'intérieur d'une théologie conçue comme une science pratique" (Mette 1978: 342). De son côté, Norbert Greinacher en fait la "théorie critique de la pratique ecclésiale dans la société" (Greinacher 1990). Johannes van der Ven la présente comme une théologie empirico-critique portant sur "les actions religieuses et pastorales dans leur(s) contexte(s) ecclésiaux et sociaux" (van der Ven 1990: 240), précisant son intérêt pour les aspects structurels et fonctionnels de l'action. De même la "fundamental practical theology" de Don Browning (1991) nous paraît familière, tant dans son orientation que dans sa démarche.

Jacques Audinet (1990), un pionnier de la théologie pratique d'expression française, a récemment situé la praxéologie pastorale comme un type de théologie pratique. Il la qualifiait alors de théologie pratique fondamentale, peut-être à tort dans la mesure où sa typologie, malgré ses possibilités combinatoires, distingue ainsi la praxéologie pastorale des théologies pratiques empiriques et critiques... dont elle partage cependant les orientations et les méthodes.

On peut actuellement situer la praxéologie pastorale comme une approche de théologie pratique. Mais on doit noter quelques différences entre elles, malgré leur objet commun, leurs connivences herméneutiques et méthodologiques. Par exemple, la théologie pratique a une histoire relativement longue, des maitres, des textes fondateurs théologiques et philosophiques aujourd'hui classiques (pensons à Schleiermacher, si présent en Europe). La praxéologie pastorale est beaucoup plus récente, ses textes fondateurs relèvent tout autant d'autres sciences que de la théologie et de la philosophie. Enfin, les sciences de l'action lui servant de référent méthodologique, son orientation est dès l'origine résolument praxique aussi bien que critique. 


\section{Le parcours de praxéologie pastorale}

La praxéologie pastorale vise l'élaboration de pratiques conscientes de leurs enjeux et de leur fonctionnement; promotrices des responsabilités de leurs divers acteurs; capables de se dire, d'établir leur cohérence et leur pertinence quant à leur culture et à la tradition chrétienne; soucieuses enfin de leur efficacité. A cet effet, et d'autant qu'elle se situe dans un contexte de formation pastorale, elle a longuement travaillé à l'élaboration de sa méthode; en témoigne son questionnaire-cadre en révision constante depuis 20 ans (Nadeau et Raymond 1987). Nous la présenterons, dans la foulée de Lonergan, comme un cadre destiné à favoriser la créativité et la collaboration [... à mettre] en relief les divers groupes d'opération que les théologiens doivent accomplir pour s'acquitter de leurs différentes tâches" (Lonergan 1978: 9). Ainsi, le parcours de praxéologie pastorale se module sur cinq niveaux interactifs où on retrouve les dynamiques de la connaissance, qui sont en fait des dynamiques pratiques, que Lonergan désigne comme attending, understanding, judaing, deciding, acting.

\subsection{L'observation}

Au point de départ, se situe une pratique particulière, souvent une pratique qui pose question ou que l'on vise à améliorer. L'observation y opère une première distance, une première "objectivation" souvent désinstallante (Grand'Maison 1973:34; 1974: 6-9; Nadeau 1987e:106). Elle vise à dégager et à analyser les éléments-clefs de la pratique, à en identifier les forces et les faiblesses.

Bien qu'elle s'ouvre avec le "récit spontané" que l'acteur fait de sa pratique, cette étape vise l'observation et l'analyse systématique d'une pratique selon des approches qui relèvent tantôt de l'analyse sémantique et pragmatique, tantôt de l'analyse existentielle. Confrontant l'agent aux faits et aux interrelations de sa pratique, l'observation lui permet d'en corriger une perception souvent naïve. Une de ses approches sémantiques distingue et met en relation les pôles structurels d'une pratique par le biais des questions de base de l'analytique de l'action: "qui, quoi, où, quand, comment, pourquoi" (Ricoeur 1977a, 1983: 88s; Raymond 1987a). Sont ainsi pris en considération les acteurs de la pratique, ses enjeux et ses intérêts, ses relations au milieu socio-culturel et ecclésial, son rapport au temps et à 
l'histoire, ses modes de communication, d'élaboration et de réalisation, ou ses effets, etc.

Cette observation se nourrit surtout de techniques qualitatives telles le verbatim rédigé de mémoire, la carte de relations, l'entrevue non dirigée, etc. Des explorations d'ordre quantitatif permettent de compléter le tableau, particulièrement quant au milieu. De ce premier panorama, l'agent fait enfin ressortir les forces et faiblesses, les problèmes de la pratique dont il poursuivra plus loin l'exploration.

Le regard attentif et l'écoute active constituent les chevilles ouvrières de cette étape. La découverte du sens et des enjeux d'une pratique ne relève pas seulement d'une démarche de réflexion, mais d'abord et avant tout de l'observation de la pratique et de la prise en compte des discours de ses acteurs. Les pratiques, en effet, ne sont pas des événements naturels, mais des événements historiques façonnés par des humains avec leurs intérêts, leurs projets, leur culture, etc. Cette étape fonde l'interprétation théologique qui n'est pas une affaire concernant seulement l'intelligence ou la foi du théologien ou du ministre, mais bien celles de l'ensemble des acteurs de la pratique. D'où l'importance fondamentale de l'attention à ce qui se vit et se dit dans les pratiques.

Les premiers critères de validité de la praxéologie pastorale relèvent donc des critères d'une observation scientifique. C'est à eux qu'elle se confronte d'abord, tout en sachant que l'observation relève elle-même de la pratique et que toute grille d'observation est elle-même située et "chargée". C'est pourquoi j'aime à parler des "prises" tout autant que des "données" de l'observation. D'où notre saisie de l'observation comme élaboration et notre prise en compte du monde de l'observateur.

Une des approches existentielles que nous avons privilégiée interroge la pratique quant à ce que nous identifions comme les cing fonctions d'élaboration des pratiques: élaboration du sens des réalités, de l'identité des personnes, du rapport à l'Autre, des collectivités, et enfin de l'éthique (Greeley 1972; Comité Catholique 1975, 1980; Nadeau 1987a,1987c). Ces fonctions désignent pour nous des dimensions fondamentales de l'expérience humaine présentes à toute pratique dont elles déterminent les drames et les enjeux. Les pratiques pastorales qui se veulent pratiques de salut doivent y être particulièrement sensibles, de même que la praxéologie pastorale qui les étudie. 
L'interprétation reviendra sur ces fonctions. Qu'est-ce que l'Évangile en effet sinon des femmes et des hommes qui retrouvent dignité et sens à leur vie, découvrent ou redécouvrent le visage de Dieu, se redressent et en témoignent, forment communauté, réélaborent leurs rapports éthiques? C'est bien dans l'une ou l'autre de ces dimensions que les contemporains de Jésus ont fait l'expérience du salut, qu'ils l'ont reconnu ou rejeté.

\subsection{La problématisation}

La problématisation vise à relier les données de l'observation en spécifiant les relations entre les termes de la pratique, particulièrement ceux qui font problème, de façon à en dégager soit un diagnostic, soit une dramatique fondamentale. Il s'agit de retisser la configuration ou le "réseau d'intersignification" (Ricoeur) de la pratique que l'analyse précédente a en quelque sorte défaits. Déjà présentes à l'étape précédente par leurs techniques d'observation, les sciences sociales tiennent ici un autre rôle. Selon le type de problèmes exhumés par l'observation, le chercheur recourra aux diverses expertises des sciences humaines pour y choisir ou élaborer à partir d'elles un modèle dialectique, historique, analogique, etc. (Nadeau 1987b)- de compréhension de la pratique. En proposant et critiquant diverses hypothèses de sens dans un nécessaire dialogue avec les sciences humaines, le chercheur ou l'acteur tente de saisir et de mettre en perspective, socio-culturelle et religieuse, la configuration des drames et des enjeux de la pratique, déterminant ainsi ce à quoi l'interprétation sera sensible dans sa relecture de la tradition.

\subsection{L'Interprétation théologique}

La première étape se centrait sur la pratique que la seconde situait dans l'intelligence de sa culture. Celle-ci met en corrélations mutuellement critiques (Lucier 1972) la tradition chrétienne et la dramatique de la pratique. Une double circulation herméneutique y vise l'apport de la tradition chrétienne à la pratique et celui de la pratique à la dynamique chrétienne. Il s'agit ainsi de clarifier, critiquer, réviser les options théologiques effectives de la pratique en regard de l'expérience contemporaine (processus commencé avec la problématisation) et de la tradition chrétienne; d'établir et de critiquer, mais surtout de stimuler la qualité évangélique de la pratique. 
Les pratiques, comme la tradition chrétienne, mettent en jeu et proposent une pluralité d'interprétations, un certain nombre de possibilités d'être historiques ${ }^{3}$. D'où le souci d'une herméneutique capable de dégager l'interprétation ou la métaphore (sans doute pas la même pour tous) qui, répondant le mieux aux données ou aux prises de la tradition et de la pratique, s'avère la plus salutaire pour ses acteurs et ses "lecteurs".

Le premier temps de cette étape permet à l'agent d'exprimer les corrélations qu'il établit spontanément entre sa pratique et la tradition chrétienne. Le second vise une herméneutique de la tradition chrétienne, d'abord centrée sur l'Écriture. Nous avons fait le pari d'y lire d'abord des récits de façon à en tirer des enjeux, des dramatiques, des dynamiques praxiques (Ricoeur 1983: 85-129), tout en soulignant les rapports entre temporalité, pratique et récit (Ricoeur 1983, 1990). Cette première rencontre systématique avec l'Écriture est donc davantage particulière que globale, analytique que synthétique. II ne s'agit pas d'appliquer une synthèse théologique ou biblique sur la pratique en vue de l'évaluer selon une approche normative, mais d'abord de rencontrer des drames semblables dans la pratique de Jésus, dans les praxein apostolón (titre particulièrement évocateur pour notre propos) et dans celles d'Israël. Suivent des lectures praxéologiques et interprétatives de pratiques ecclésiales, de déclarations du Magistère et de discours théologiques qui semblent pertinents à la saisie et au dénouement du drame de la pratique.

Des corrélations, des oppositions, des intuitions nouvelles émergent de telles lectures. Les derniers moments de l'interprétation visent à les déployer en un discours cohérent en rapport avec la dynamique fondamentale du mystère chrétien, de façon à en tirer des interpellations concrètes et fécondes pour la pratique.

Enfin, l'interprétation théologique s'applique particulièrement à saisir la fonction révélante des pratiques (Ladrière 1973; Ricoeur 1977b; Nadeau 1993). II en va du caractère des pratiques pastorales d'être pratiques de révélation, à tout le moins "disclosure situations" (Ramsey). C'est-à-dire non seulement de prendre soin du monde mais de projeter un monde où un Autre se tient avec nous. Telle l'oeuvre à laquelle nous avons rapidement analogué les pratiques, ce n'est que

3 Le travail de Gilles Raymond sur la guérison de l'aveugle-né en Jean 9 met bien en lumière cette dynamique (Raymond $1987 \mathrm{~b}$ et 1989). 
lorsqu'elles sont ainsi lues et comprises et qu'une telle compréhension conduit à une appropriation des possibilités ouvertes par elles, qu'on peut considérer les pratiques pastorales comme "complétées" (Capps 1984: 47), c'est-à-dire effectivement signifiantes quant à leur fonction révélante Par exemple, si les pratiques énumérées en Mt 25,31-46 sont loin d'être insignifiantes au moment où elles se produisent, on doit reconnaître que le jugement du Fils de l'homme en révèle la nature profonde, qu'il les complète en quelque sorte. Le temps de l'interprétation se fait alors temps, performatif, de l'attestation aussi bien que de l'interpellation de la collaboration du divin et de l'humain.

Si certains aspects du salut peuvent être objets d'analyse des sciences sociales, celui-ci relève en propre de l'interprétation théologique. La praxéologie pastorale s'efforce donc, non seulement d'analyser et de critiquer les pratiques aux plans précédemment suggérés, mais d'en dégager les virtualités et les prolepses de salut, à travers la mise au jour des mondes ouverts par les pratiques et la saisie de leurs processus d'élaboration, d'inscription et de manifestation croyantes. De là pourront surgir de nouvelles voies d'action pour bâtir et habiter ce monde. Une telle approche pourrait peut-être commencer à spécifier un rapport praxéologique à la révélation.

\subsection{La réélaboration opérationnelle}

La réélaboration opérationnelle de la pratique marque le moment de la délibération et de la décision devant des choix pratiques. Si la praxéologie pastorale s'approche par certains aspects d'une recherche évaluative (Nadeau et Foucault 1992), voici le moment d'établir l'utilité de son parcours (Patton 1980: 282). Cette étape vise à dégager les effets de l'interprétation sur la pratique, de façon à choisir, planifier et gérer une intervention sur la pratique ou le milieu, qui soit responsable, praticable et recevable, c'est-à-dire attentive aux différents acteurs de la pratique, à leurs ressources, à leurs possibilités et à celles du milieu. Trois conditions sont nécessaires pour mener à bien une telle intervention: une information valide, un choix libre et informé par des cartes cognitives et des objectifs, un engagement interne (Argyris 1970: 16-20). Les étapes précédentes ont permis de dégager l'information et de choisir ou d'élaborer les schémas de compréhension nécessaires à la saisie de la pratique. Voici venu le temps de repréciser un engagement, d'en rajuster le tir, d'y arrimer à neuf des moyens. 
Trop de pratiques pastorales n'ayant que des objectifs fondamentaux ou généraux dont on ne peut évaluer la réalisation, on veillera à spécifier quelques objecfifs qui s'avéreront d'autant plus motivants qu'ils seront susceptibles d'être atteints à plus ou moins court terme. Les dimensions communicatives et opérationnelles des pratiques telles l'animation, la participation, l'information et la formation, l'orientation, la concertation, la planification, la gestion, l'évaluation (Nadeau et Raymond 1987: 304-306), déjà prises en compte lors de l'observation, trouvent ici une occasion d'ajustement et de relance.

En tenant compte des rétroactions et des résistances prévisibles, on élaborera enfin un plan d'action concret où figureront, dans une optique d'intervention planifiée, les six pôles qui ont déjà guidé l'observation: objectifs, acteurs destinataires et ministres, étapes, opérations, responsabilités, échéances, objets et modes d'évaluation, etc.

\subsection{La prospective et l'évaluation}

Bien que nous la présentions en dernier lieu, la prospective marque en fait l'ensemble de la démarche (Lucier 1973; Nadeau 1987d). Par exemple, elle orientait déjà notre rapport aux sciences sociales et aux sciences de l'action dont le recours situait pratiquement la praxéologie pastorale sous leur autorité. Mais cette autorité était ellemême subordonnée à une intentionnalité, à une prospective: il s'agissait de voir pour, d'écouter pour, d'interroger pour, etc. Déjà, les sciences de l'organisation et la recherche évaluative sont claires sur ce point (Douglas 1976: 3; Patton 1980: 269-282; Nadeau et Foucault 1992).

Technique, la prospective vise à dégager les possibilités et les suites de l'intervention prévisibles à moyen et à long termes. Poétique, elle renvoie au coeur de la pratique dont elle nomme la mémoire propre, l'espérance, l'horizon. Certains y vivent une démarche spirituelle où, en la présence de l'Autre, l'agent se recentre sur ce qui le fait vivre et qui l'appelle à s'engager dans cette pratique, nomme à neuf sa foi et son espérance, réaffirme sa responsabilité face à cette Parole qui l'interpelle du coeur de l'agir, de l'Écriture, de l'histoire et de la communauté.

Quant à l'évaluation, signalons simplement qu'elle ne porte pas ici sur la pratique, la praxéologie pastorale relevant à maints égards d'une 
démarche évaluative, mais sur le parcours praxéologique effectué, quant à ses découvertes, ses points d'ombre, ses acquis, etc.

\section{Conclusion}

Le rôle des approches scientifiques en praxéologie pastorale a régulièrement affleuré au fil des pages. D'abord quant au rapport fondamental de la praxéologie pastorale aux sciences de l'action, puis quant aux différents moments de sa démarche. Une science de l'agir pastoral ne saurait être qu'interdisciplinaire ou intradisciplinaire (Lefebvre 1971; Grand'Maison 1974; van der Ven 1992) si elle veut répondre à la complexité et aux requêtes des pratiques ainsi qu'à l'intelligence de notre temps.

D'une certaine façon, ce rapport soumet la praxéologie pastorale à l'autorité de ces approches empiriques, aussi bien qu'à celle de la foi chrétienne. Mais si cette autorité empirique consiste d'abord dans l'exigence de s'arrêter pour voir, écouter, interroger, elle rejoint quelque chose de l'Evangile lui-même (Grand'Maison 1973: 35-38). Cette autorité, d'abord méthodologique, n'est cependant pas sans risque comme l'ont signalé plusieurs, soulignant par exemple les effets sécularisants de leur paradigme de base (Garceau 1989). Ainsi il nous semble parfois que nos approches de théologie pratique font peu de place à un dire la révélation. D'où la nécessité d'une philosophie et d'une herméneutique qui puissent resituer l'articulation des approches scientifiques et des discours de révélation. Certains $y$ ont vu un paradoxe épistémologique majeur qu'ils exprimaient ainsi: "alors que la théologie pastorale ${ }^{4}$ qui s'oriente dans cette voie [de l'efficacité] adopte implicitement et explicitement les critères de scientificité et de validation des sciences de la nature, elle semble aussi avoir développé un large consensus sur l'utilisation du modèle herméneutique au coeur même de sa démarche" (Fortin-Melkevik et Keating 1992: 372).

On ne peut éviter la question, bien que je ne puisse la travailler ici. On pourra toutefois se demander si les critères visés sont bien ceux des sciences de la nature. De plus, il nous paraît difficile de mener une recherche empirique sans prendre en compte son cadre herméneutique et

4 Le contexte de leur intervention porte à croire que les auteurs désignent ainsi ce que nous avons désigné comme théologie pratique. 
la dimension herméneutique du réel humain lui-même. Par ailleurs, il nous semble que notre objet, les pratiques considérées comme actions sensées, ne nous laisse guère le choix de tenter une telle articulation. En témoigne, parmi d'autres, cette affirmation d'Habermas (1986: 44): "Toute expression dotée de sens peut être identifiée sur deux plans: en tant qu'événement observable, mais aussi en tant que signification objectivée susceptible d'être comprise." Se déploie alors l'arc herméneutique explication-compréhension où l'analyse est un passage nécessaire entre interprétation naïve et interprétation érudite, interprétation de surface et interprétation en profondeur, allant de $\underline{c e}$ que dit le texte (ou l'action) à ce sur quoi il parle, la sorte de monde qu'il ouvre, ce qui en lui vise un monde possible (Ricoeur 1971).

Ainsi sommes-nous conviés, sinon forcés, à la complexe et exigeante articulation entre engagement croyant, sciences humaines et sociales, éthique, philosophie, théologie. La praxéologie pastorale, comme la théologie pratique actuelle, relève de cet effort dont l'expérience a mené plusieurs intervenants pastoraux ou étudiants en théologie à une conversion au réel des pratiques, à l'altérité et à la responsabilité de leurs divers sujets, à une ouverture sur un monde différent. En d'autres mots: à "un profond renouvellement du regard, du discernement, du geste et de l'horizon de sens et de vie qui qualifient une tout autre pratique de foi en prise sur un profond changement culturel et social à opérer" (Grand'Maison 1987:7).

Dans le cadre de cette publication, on soulignera enfin que si le modèle praxéologique en appelle aux possibilités de collaboration interdisciplinaire de l'université, il met aussi en jeu sa fonction sociale, par exemple dans l'articulation de ses tâches de recherche, de critique, de formation professionnelle (un des vecteurs majeurs de la praxéologie pastorale même si notre exposé a peu insisté sur celle-ci), voire d'intervention sociale. "Lieu de distance critique et de recherche systématique et théorique, l'université n'en est pas moins le lieu de formation de gens qui seront, non pas d'abord des 'universitaires', mais essentiellement et majoritairement des intervenants sociaux de tous ordres" (Lucier 1973: 29), parmi lesquels se situent les intervenants pastoraux dont la formation doit au moins équivaloir celle des autres intervenants. 


\section{BIBLIOGRAPHIE}

ARGYRIS, Chris. 1970. Intervention Theory and Methed. Reading (Mass) - Don Mills (Ont), Addison-Wesley.

AUDINET, Jacques. 1990. "Diversité des théologies pratiques", Revue de l'Institut catholique de Paris 33 (janvier-mars): 75-96.

BROWNING, Don S. 1991. A Fundamental Practical Theology. Minneapolis, Augsburg Fortress.

CAPPS, Donald. 1984. Pastoral Care and Hermeneutics. Philadeiphie, Fortress Press.

COMITE CATHOLIQUE DU CONSEIL SUPERIEUR DE L'EDUCATION. 1975. Voies et impasses 1. Dimension religieuse du projet scolaire. Québec, Ministère de l'éducation.

COMITE CATHOLIQUE DU CONSEIL SUPÉRIEUR DE L'EDUCATION. 1980. Voies et impasses 5. L'animation pastorale. Québec, Ministère de l'éducation.

DAVAL, Roger. 1963. "La praxéologie", Sociologie du travail 2 (avril): $135-155$.

ESPINAS. 1897. Les origines de la technolegie. Paris, Alcan.

FARLEY, Edward. 1983a. Iheologia. The Eragmentation and Unity of Theological_Education. Philadelphia, Fortress.

FARLEY, Edward.1983b. "Theology and Practice Outside the Clerical Paradigm", dans Practical Theology. (BROWNING, D.S., éd.), San Francisco, Harper and Row, 21-41.

FARLEY, Edward.1987. "Interpreting Situations: An Inqury into the Nature of Practical Theology", dans Eermation and Reflection. (MUDGE L.S. et J.N. POLING., dir.), Philadelphia, Fortress Press, 1-26.

FORTIN-MELKEVIK, Anne et Bernard KEATING,. 1992. "Quelle autorité pour les approches empiriques en théologie?", dans Les aporoches empiriques en théologie. Empirical Approaches in Theologr. (PELCHAT, M., éd.), Université Laval, Faculté de théologie (Théologies pratiques \#4), 371-378.

GARCEAU, Benoît. 1989. "Les défis des sciences humaines pour l'intelligence de la foi", dans Défis présents et à venir de l'université catholique. (CROTEAU, J., éd.), Ottawa, Université Saint-Paul, 139-166. 
Jean-Guy Nadeau: La praxéologie pastorale...

GOYETTE, Gabriel et Michelle LESSARD-HEBERT .1987. La rechercheaction. ses fonctions. ses fondements et son instrumentation. Québec, Presses de l'Université du Québec.

GRAND'MAISON, Jacques. 1973. La seconde évangélisation. Tome 2.1 Outils majeurs. Montréal, Fides, (Héritage et projet \#2); le chapitre ici cité: "Science, art et Évangile du regard" a été réédité dans La praxéologie pastorale_le (NADEAU, J.-G., éd.), Montréal, Fides, (Cahiers d'Études pastorales \#4), 71-90.

GRAND'MAISON, Jacques.1974. "La carte des relations et son défi épistémologique", Études pastorales '74. Université de Montréal: 6-25; réédité dans La praxéologie pastorale _ـ (NADEAU, J.-G., éd.), Montréal, Fides, (Cahiers d'Études pastorales \#4), 127148.

GRAND'MAISON, Jacques. 1975. Des milieux de travail à réinventer. Montréal, Presses de l'Université de Montréal.

GRAND'MAISON, Jacques. 1987. "Ouverture", dans " La praxéologie pastorale L (NADEAU, J.-G., éd.), Montréal, Fides, (Cahiers d'Études pastorales \#4), 7-9.

GREELEY, Andrew. 1972. Unsecular Man. The Persistence of Religion. New York, Schoken Press.

GREINACHER, Norbert. 1990. "La théologie pratique en tant que théorie critique de la pratique ecclésiale dans la société", dans Les Études pastorales à l'université - Pastoral Studies in a University Setting. (VISSCHER, A., éd.), Ottawa, Presses de l'Université d'Ottawa, 279-297.

HABERMAS, Jürgen. 1986 (1983). Morale et communication. Paris, Cerf.

JERVOLINO, Domenico. 1991. "Herméneutique de la praxis et éthique de la libération", dans Paul Ricoeur. Les métamorphoses de la raison herméneutique. (GREICH, J. et R. KEARNY, éd.), Paris, Cerf, 223230.

KAUFMANN, Arnold. 1968. L'homme d'action et la science. Introduction élémentaire à la praxéologie. Paris, Hachette.

KOTARBINSKI, Tadeusz.1937. "Idée de la méthodologie générale Praxéologie", Actualités scientifiques et industrielles 533.

KOTARBINSKI, Tadeusz.1965. Praxiology. An Introduction to the Sciences of Efficient Action. Oxford, Pergamon.

LADRIERE, Jean. 1984 (1973). "La performativité du langage liturgique", dans L'articulation du sens 1 . Paris, Cerf, (Cogitatio Fidei \#125), 55-65. 
LAMB, Matthew. 1976. "The Theory-Praxis Relationship in Contemporary Theology", CISA Proceedings 31: 149-178; repris et révisé dans LAMB, M. 1982. Solidarity with Victims. New York, Crossroads,, 61-99.

LEFEBVRE, Marcel. 1971. "Vers une nouvelle approche de la théologie pastorale", Nouvelle revue théologique 93: 29-49.

LONERGAN, Bernard. 1978 (1972). Pour une méthode en théologie. Montréal, Fides, (Héritage et projet \#20).

LUCIER, Pierre. 1972. "Réflexions sur la méthode en théologie", Sciences Religieuses II/1: 63-75; réédité dans La praxéologie pastorale 2. (NADEAU, J.-G., éd.), Montréal, Fides, (Cahiers d'Études pastorales \#5), 61-77.

LUCIER, Pierre. 1973. "Un modèle d'analyse du geste d'intervention pastorale",dans Etudes pastorales '73. Faculté de théologie, Université de Montréal, 7-35.

LUCIER, Pierre. 1974. "Théologie et praxéologie", dans Etudes pastorales '74. Faculté de théologie, Université de Montréal, 2740; repris dans La praxéologie pastorale L (NADEAU, J.-G., éd.), Montréal, Fides, (Cahiers d'Études pastorales \#4), 15-31.

METTE, Norbert. 1978. Theorie der Praxis. Düsseldorf, Patmos Verlag. METTE, Norbert. 1979. "Praktische Theologie as Handlungswissenschaft", Diakonia 10: 190-203.

von MISES, Ludwig. 1950. Human Action. A Treatise on Economics. New Haven, Yale University Press.

NADEAU, Jean-Guy. 1987a. "La vocation et la mission des baptisés", Prêtre et pasteur. Revue des agents de pastorale vol 90 no 8 (septembre): $450-458$.

NADEAU, Jean-Guy. 1987b. "La problématisation en praxéologie pastorale", dans La praxéologie pastorale L. (NADEAU, J.-G., éd.), Montréal, Fides, (Cahiers d'Études pastorales \#4), 181 206.

NADEAU, Jean-Guy. 1987c. "Les cinq fonctions d'élaboration des pratiques", dans La praxéologie pastorale 2. (NADEAU, J.-G., éd.), Montréal, Fides, (Cahiers d'Études pastorales \#5), 79-86.

NADEAU, Jean-Guy. 1987d. "La prospective en praxéologie pastorale", dans La praxéologie pastorale 2. (NADEAU, J.-G., éd.), Montréal, Fides, (Cahiers d'Études pastorales \#5), 262-270.

NADEAU, Jean-Guy. 1987e. "Les agents de pastorale et l'observation du réel", dans La praxéologie pastorale L (NADEAU, J.-G., éd.), Montréal, Fides, (Cahiers d'Études pastorales \# 4), 91-106. 
Jean-Guy Nadeau: La praxéologie pastorale...

NADEAU, Jean-Guy. 1990. "Pour une science de l'action pastorale. Le souci de la pertinence pratique", dans Les Etudes pastorales à l'université - Pastoral Studies in a University Setting. (VISSCHER, A., éd.), Ottawa, Presses de I'Université d'Ottawa, $136-150$.

NADEAU, Jean-Guy. 1993 "La fonction révélante des pratiques pastorales", à paraître avec les Actes du colloque international de théologie pratique tenu à Lausanne en mai 1992, sous la direction de Bernard Reymond.

NADEAU, Jean-Guy et Hélène FOUCAULT. 1992. "Évaluation pastorale, recherche évaluative et autorité des approches empiriques en théologie pratique", dans Les approches empiriques en théologie. Empirical Approaches in Theology. (PELCHAT, M., éd.), Université Laval, Faculté de théologie, (Théologies pratiques \#4), 51-78.

NADEAU, Jean-Guy et Gilles RAYMOND. 1987. "Dossier de travail en praxéologie pastorale", dans La praxéologie pastorale 2. (NADEAU, J.-G., éd.), Montréal, Fides, (Cahiers d'Études pastorales \#5), 288-293.

OGLETREE, Thomas W. 1983. "Dimensions of Practical Theology: Meaning, Action, Self", dans Practical Theology. (BROWNING, D.S.,éd.), San Francisco, Harper and Row, 83-101.

PATTON, Michael Quinn. 1980. Qualitative Evaluation Methods. Beverly Hills/London, Sage.

RAYMOND, Gilles. 1987. "Les six pôles d'exploration d'une pratique", dans La praxéologie pastorale1. (NADEAU, J.-G, éd.), Montréal, Fides, (Cahiers d'Études pastorale \# 4), 107-126

RAYMOND, Gilles. 1989. "Des interprétations qui aveuglent ou illuminent", dans L'interprétation. un défi de l'action pastorale. (NADEAU, J.-G., éd.), Montréal, Fides, (Cahiers d'Études pastorales \#6), 135-149.

RICOEUR, Paul. 1971. "The Model of the Text: Meaningful Action Considered as a Text", Social Research 38: 529-555.

RICOEUR, Paul. 1977a. "Le discours de l'action", dans RICOEUR, P. et al., La sémantique de l'action. Paris, CNRS, 3-137.

RICOEUR, Paul. 1977b. "Herméneutique de l'idée de Révélation", dans RICOEUR, $P$. et al. La révélation. Bruxelles, Facultés universitaires Saint-Louis, 15-54.

RICOEUR, Paul. 1983. Temps et récit. T. 1, Paris, Seuil. 
RICOEUR, Paul. 1989a. "Les structures téléologique et déontologique de l'action: Aristote et/ou Kant?", dans Etudes pasterales ef interprétation. (NADEAU J.-G., éd.), Montréal, Fides, (Cahiers d'Études pastorales \#6), 15-27.

RICOEUR, Paul. 1989b. "Praxéologie pastorale, herméneutique et identité", dans Etudes pastorales et interprétation (NADEAU, J.G., éd.), Montréal, Fides, (Cahiers d'Études pastorales \#6), 265275.

RICOEUR, Paul. 1990. Soi-même comme un autre. Paris, Seuil.

SCANLON, Michael J. 1988. “Language and Praxis: Recent Theological Trends", Catholic Theological Society of America Proceedings 43: 80-89.

SHEEHAN, Mary Ellen. 1984. "Theological Reflection and Theory-Praxis Integration - An Experience with the Case Study Method", Sciences pastorales - Pastoral Sciences 3: 25-38.

TRACY, David. 1975. Blessed Rage for Order. New York, Seabury.

TRACY, David. 1983. "The Foundations of practical theology", dans Practical Theology. (BROWNING, D.S., éd.), San Francisco, Harper and Row, 61-82.

van der VEN, Johannes A. 1988. "Practical Theology: from Applied to Empirical Theology ", Journal of Empirical Theology vol 1 no 1: 7-27.

van der VEN, Johannes A. 1990. “Practical Theology as CriticalEmpirical Theology", dans Les Études pastorales à ل'université Pastoral Studies in a University Setting. (VISSCHER, A., éd.), Ottawa, Presses de l'Université d'Ottawa, 238-262.

van der VEN, Johannes A. 1992. "Congregational Studies from the Perspective of Empirical Theology", dans Les approches empiriques en théologie. Empirical Approaches in Theologr. (PELCHAT, M., éd.), Université Laval, Faculté de théologie, (Théologies pratiques \#4), 101-130. 